\title{
Hypertrophic cardiomyopathy: obstruction flushed out with exercise echocardiography
}

\author{
Stella MARCHETTA, MD; Marie MOONEN, MD, PhD; Raluca DULGHERU, MD; \\ Patrizio LANCELLOTTI, MD, PhD; Luc PIÉRARD, MD, PhD \\ ${ }^{1}$ University of Liège Hospital, GIGA Cardiovascular Sciences, Dept. of Cardiology, Heart Valve Clinic, University Hospital Sart Tilman, Liège, \\ Belgium.
}

Keywords Hypertrophic cardiomyopathy - dynamic subaortic obstruction - exercise.

We describe the case of a 17 -year-old girl with a recent diagnosis of hypertrophic cardiomyopathy. Her main symptom was post-exercise dizziness without loss of consciousness. Two-dimensional transthoracic echocardiography revealed marked hypertrophy of the basal segment of the interventricular septum $(27 \mathrm{~mm}$ ) (figure 1, panel A) with no subaortic gradient at rest and during a Valsalva manoeuvre performed in the supine position (figure 1, panel B). The additional investigations did not reveal sustained or non-sustained ventricular tachycardia on 48-hour Holter monitoring nor hypotension during bicycle exercise testing. The patient underwent exercise echocardiography in a semi-supine position on a tilting exercise table. Echocardiographic imaging was continuous during exercise and recovery and did not reveal left ventricular outflow tract obstruction (LVOTO) (figure 1, panels $\mathrm{C}$ and D). Episodes of dizziness persisted; exercise echocardiography was repeated. Shortly after exercise cessation, the patient was placed in the upright position. A rapid increase of the velocity in the LV outflow tract was identified, corresponding to a 70-mm Hg peak subaortic pressure gradient (figure 1, panel E), accompanied by lightheadedness and profuse diaphoresis. This orthostatic manoeuvre mimics real-life haemodynamics by reducing venous return and preload while LV contractility remains increased. The phenomenon reduces the diameter of the left ventricle, and unmasks systolic anterior motion of mitral valve and LVOTO. The presence of significant septal hypertrophy in a symptomatic patient without subaortic obstruction at rest should prompt the search of a dynamic LVOTO not only during exercise, but also after exercise cessation, in the standing position. 

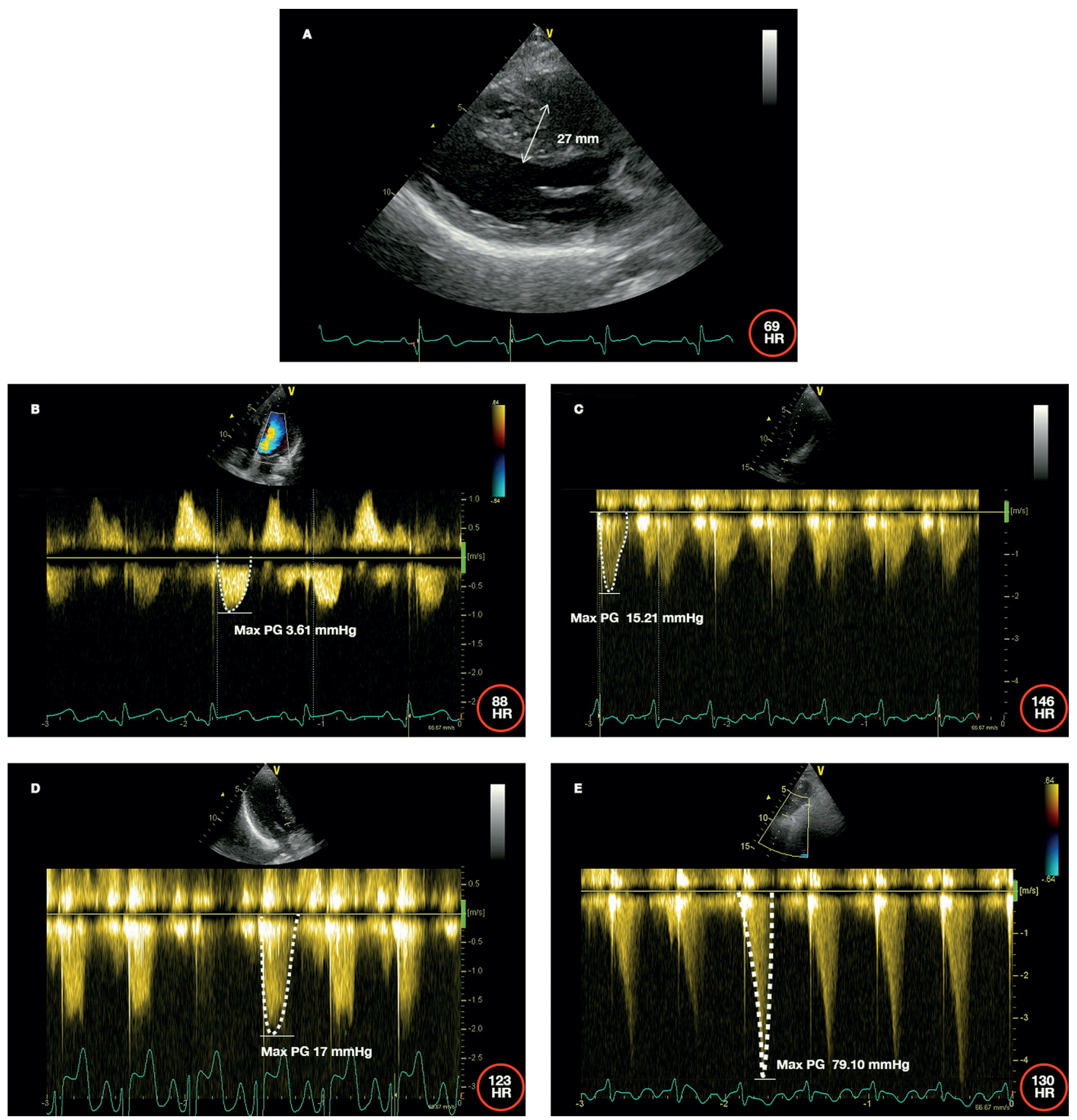

Fig. 1 (A) Interventricular septum, (B) Subaortic gradient at rest, (C) Subaortic gradient during peak exercise, (D) Subaortic gradient after exercise in supine position, (E) Subaortic gradient after exercise in upright position. 